\title{
Research on the Construction of Chinese and Russian Rock Culture Heritage Database in Heilongjiang Basin
}

\author{
Liu Yang ${ }^{*}$ Xu Ying \\ Heihe University Heihe Heilongjiang 164300
}

Keywords: Rock paintings; Yellow River basin; Database

\begin{abstract}
On the basis of analyzing the necessity of protecting the cultural heritage of rock paintings in the Yellow River Basin, this paper mainly expounds the process of building the database of rock paintings, and looks forward to the protection of rock paintings from the following four aspects: the application of new technology for protecting rock paintings, the legislation for protecting rock paintings, the publication of rock paintings and the cooperation of rock paintings protection agencies.
\end{abstract}

\section{Introduction}

The vast area of Heilongjiang River Basin is one of the most important distribution areas of rock paintings in China, and it is also a master of rock paintings in northern China. Rock paintings in the Yellow River Basin have become one of the great roots of Chinese civilization with its magnificent quantity, dense concentration and wonderful content. But the most brilliant part of Chinese civilization is suffering from irreversible human destruction and natural erosion. Since the late 1980s, people have come to realize the value of rock paintings; some people began to grasp the plan, purposefully. Most of the rock paintings have no protection against wind, rain and natural weathering; the air pollution and acid rain caused by industrial waste gas accelerate the weathering process of rock paintings. Rock paintings, like other cultural relics, cannot be regenerated, destroying one or less. In this case, how to protect the rock paintings, so as to extend their life, has become an urgent need to solve the major issue. In recent years, with the deepening of the study of rock paintings in the Yellow River Basin and the enhancement of people's awareness of protection, rock paintings protection has made great progress.

\section{Database Construction of the Yellow River Rock Culture Heritage}

At present, characters, pictures, audio recordings, video recordings, digital multimedia and so on are the basic ways of cultural heritage preservation. The comprehensive use of various ways makes the information of the protected objects be truly, comprehensively and systematically recorded and preserved. For rock paintings, there are three ways to preserve them: documents that record text and table information, rubbings containing picture information, line drawing and digital photos, and rock paintings database. Because of the different storage carriers, documents, rubbings and line tracing, digital photos, database, the difficulty of preservation and the preservation effect of the 
three are generally negatively correlated. Documents, rubbings and line traces are the most difficult to preserve, and their paper carriers require higher environmental conditions such as temperature and humidity. In particular, the Xuan paper used for rubbing is extremely vulnerable to pests and moisture. The best preservation is rock painting database based on various storage devices. Firstly, the existing storage devices are small in size and save space; secondly, the reproducibility of the database improves the security of the rock painting files; thirdly, the storage devices require less environmental conditions, and generally do not need special maintenance. It can be said that rock art database is the most durable, safest and most reliable way of preservation at present. Generally speaking, the rock painting database is a collection of rock painting archives data organized in a specific way, with automatic query, modification, information retrieval, matching, output and other functions, not only the digital conversion of rock painting archives.

\subsection{Description of Non Image Data of Rock Paintings}

Non image data include texts and tables related to rock art information. In the text description of rock painting image, we should pay attention to effectively combine the content of rock painting image and the external features of rock painting image. Specifically, the following image attributes should be recorded. (1) making technology: grinding, cutting, percussion, direct chisel, indirect chisel, blunt hammer, hammering hammer and carving. (2) visualization: easy, difficult and vague. (3) location: obvious, hidden, combined, repeated coverage, prominent. (4) uses: clan tribe, reproductive worship, hunting, witchcraft, migration, sacrifice, war, etc. (5) size. (6) the location of existence. (7) Image types: characters, animals and plants (tigers, leopards, wolves, deer, sheep, cattle, horses, camels, grain spikes, etc.), celestial bodies (sun, moon, stars, clouds, etc.), symbols.

\subsection{Editing and Storage of Rock Picture Data}

After collecting the required rock painting images, the most important task is image editing to meet the requirements of image storage. In the aspect of image editing, Adobe Photoshop software can process digital images with the functions of image adjustment and layer adjustment. Digital images are usually scanned with the highest resolution of 200 dots per inch. The size of digital archives of rock paintings images is usually about $600 \mathrm{x} 1000$, and each image file is about 1.5M. Rock picture can not only be added to the database, but also can be updated or deleted.

\subsection{Retrieval of Rock Art Data}

Because unstructured data such as rock paintings account for a considerable part, text-based image retrieval technology (TBIR) is more suitable for rock paintings image retrieval. In the text description of rock paintings, we should pay attention to the effective combination of image elements, shapes and other rock paintings image content and size, type, name and other external features of rock paintings image. The description of the content and features of the rock painting image should be accurate and concise, and then the index should be established according to the descriptive free text to realize the matching of keywords and feature marks. Specifically, the fast retrieval and output of rock painting image information should be realized by three steps: establishing the index database of rock painting image, querying the index image database and outputting the image indexing which meets the similarity threshold. The general framework of the retrieval process can be described as five steps: query, description, matching, extraction and verification (see figure). In addition, in view of the differences in descriptive texts between the builders and users of the rock painting database, on the one hand, the descriptive texts listed should be compiled according to certain rules (such as alphabetic alphabet sorting) in order to facilitate 
user queries; on the other hand, users should be widely collected through the feedback function of the retrieval system. Text usage habits, will use more frequent keywords into descriptive text, improve the retrieval efficiency of rock painting database.

\section{Prospects for the Protection of Rock Culture Heritage in the Yellow River Basin}

\subsection{Introduce High-tech Means to Protect and Reproduce Rock Paintings.}

In addition to physical and chemical protection methods, rock paintings can also be protected by high-tech means. Among them, the most mature and effective means of rock painting protection technology is to install solar energy electronic monitoring system in rock painting intensive areas. Because of the high price of video surveillance equipment, it can not be widely used, can only be concentrated in rock paintings, high research value, more easily pirated areas to implement control. The remote monitoring system is mainly composed of several probes and a server. The probes transmit the rock paintings to the server in real time. The protection can be seen by calling the server through the computer. The use of remote monitoring technology can be unattended in the case of early warning, and record illegal activities, play a post-mortem traceability, verification role, for the protection of cultural relics regulatory departments to provide help. At present, it is impossible to avoid the damage of natural diseases to rock paintings, but we can learn from foreign advanced rock paintings protection methods, so that rock paintings can be preserved for a long time, for people to see. Such as three-dimensional computer technology, fully reproduce the existing rock art, rock painting environment. In the future, when the rock paintings disappear completely, people can enjoy the rock paintings through 3D animation. This technology can not only scan the surrounding environment of rock paintings 360 degrees, provide more accurate pictures and data, but also reduce the damage caused by man-made exploration of rock paintings. Even if there are extremely destructive disasters such as earthquakes, rock paintings can be restored and reconstructed by this technology. On the basis of scientific grasp of rock painting data, how to better protect rock painting and repair the disease of rock painting "treatment" is of vital importance. Nanotechnology is expected to make some breakthroughs in this area. The surface protection of rock paintings is a very arduous task and challenge, which requires long-term field test and data acquisition.

\subsection{Speed Up the Legislative Process of Rock Painting Protection}

The most fundamental thing to protect rock paintings is to legislate. With laws and regulations, there is a basis for law enforcement, we can take necessary measures according to law, effective protection. Taking the lead of the relevant provincial departments in the Yellow River Basin, experts from various fields were convened to study and formulate the Interim Measures for the Protection of Rock Paintings in the Yellow River Basin, which were submitted to the State for approval and implementation. It is necessary to establish the guiding ideology, target principles and specific tasks for the protection of the cultural heritage of rock paintings, to refine the management measures, to clarify the responsibilities of units at all levels, commendation and reward, financial support and other content, so as to lay a foundation for strengthening the preservation, research and utilization of rock paintings archives. Through the implementation and improvement, experts are organized to draft the Regulations on the Protection of Rock Paintings in the Yellow River Basin. It is clear that the main body of the protection of rock paintings is the people's government at or above the county level where the rock paintings are located. The definition and scope of protection of rock paintings are made clear, and the supervision and protection responsibilities of the cultural relics management departments are clarified. From the aspects of formulating the protection plan of rock 
paintings, realizing the graded protection, delimiting the protection scope and constructing the control zone, the protection target is defined, the specific protection measures are formulated, the research and utilization of rock paintings are specified, and the damage behavior is punished, so that the protection of rock paintings in the Yellow River Basin can be carried out by the local law. In order to protect the rock paintings, it is everyone's duty, law and punishment. This will greatly enhance the seriousness and operability of rock paintings protection, and increase protection efforts. In addition, a document on expert evaluation of rock painting protection has been issued to make the protection requirements of rock paintings more standardized. The expert group on rock painting protection will assist the cultural departments in collecting, collating and examining rock painting materials, put forward opinions and suggestions on the relevant policies for rock painting protection, put forward constructive suggestions on the formulation of medium-term and long-term goals for rock painting protection in the Yellow River Basin and the establishment of new protection models, and undertake the selection, implementation and related research of key research topics on rock painting. Data compilation and other work.

\subsection{Systematic Collection of Important Rock Paintings}

Over the past decade, various provinces and regions in the Yellow River Basin have been actively conducting field investigations on rock paintings. They have published Gaishanlin's Yinshan Rock Painting, Inner Mongolia Rock Painting Cultural Interpretation, the large-scale literature series edited by Northern University for Nationalities, including Barley Rock Painting, Helan Mountain Rock Painting, and Yinshan Rock Painting. Heran Mountain and Beishan Rock Painting, Xu Cheng and Wei Zhong's Heran Mountain Rock Painting, Liu Yi's Goz Mountain Rock Painting, etc. These academic monographs and large-scale catalogues of rock paintings in Ningxia, Inner Mongolia, Henan area a lot of fine works, is an important achievement in the publication and protection of rock paintings. For the Yellow River rock paintings, publishing protection is far from enough. Firstly, on the one hand, the existing publications of rock paintings in the Yellow River Basin have been omitted because of the different subjective consciousness of the writers, which leads to the different selection criteria. On the other hand, the different compilation forms of various publications make readers confused to understand the rock paintings in the Yellow River Basin comprehensively and systematically. Secondly, the Yellow River Basin rock paintings throughout Qinghai, Gansu, Ningxia, Inner Mongolia, Shanxi, Henan Province and regions, at present, the focus of publishing protection mainly concentrated in Inner Mongolia and Ningxia Autonomous Region, other provinces and regions of publishing protection work is slightly weak. Therefore, in the future, in the publication and protection of rock paintings in the Yellow River Basin, we should select rock paintings according to the unified standards and compile publications in a unified form. Finally, a set of publications including Qinghai rock paintings, Gansu rock paintings, Ningxia rock paintings, Inner Mongolia rock paintings, Shanxi rock paintings and Henan rock paintings will be formed. The series of large-scale series of rock paintings in the Yellow River Basin should be published digitally at the same time, and a special subject database should be established to collect important rock paintings in the Yellow River Basin and fully display and reflect the physical characteristics of rock paintings in the Yellow River Basin. This is still unable to use scientific methods to protect the conditions of field rock paintings, for the preservation of original data can be said to be a great contribution.

\section{Conclusions}

Although various rock painting research institutes have been set up in the provinces of the Yellow River Basin, the institutions are basically independent and do not belong to each other, resulting in a great waste of 
resources. Therefore, it is imperative to strengthen exchanges and build a pluralistic cooperation mechanism. Specifically, first, we must establish and improve the organizational mechanism. The Yellow River Basin Rock Painting Research Cooperation Organization was established, with the Chinese Rock Painting Research Center as the leader and other provincial and regional rock painting research institutions as members. On the premise of complete organizational framework, we should formulate operable rules and regulations, integrate various forces, and lay a good organizational foundation for the protection of rock paintings in the Yellow River Basin. Two, we must establish and improve the interest mechanism. In the projects and activities of multi-party participation, clear their respective rights and obligations, through a reasonable distribution of interests to regulate the relationship between all parties. Three, we must establish a sound communication mechanism. Establish communication bridges between leaders and members of the cooperative organization and among members to avoid the phenomenon of work disjunction and shifting responsibility caused by the inadequate or inappropriate communication among the internal links and to improve the efficiency of cooperation. Only by building a multi-cooperation mechanism can we make substantial progress in the protection and research of rock paintings in the Yellow River Basin, organize related cultural activities, and strengthen exchanges and cooperation at home and abroad.

\section{Acknowledgment}

Fund projects: 2018 Heilongjiang Philosophy and Social Science Research Planning Project; Project Number: 18YSE615; Project Name: Sino-Russian Heilongjiang Valley Rock Painting Art Protection and Utilization Research.

\section{References}

[1] Evergreen, Wang Jinger. An Analysis of the Members'Benefit Balance Mechanism of the Digital Library Consortium: Taking Ningbo Digital Library as an Example [J]. Library Theory and Practice, 2014 (1): 27-30.

[2] Zhao Jihai. On the Realization Mechanism of Regional Information Service Equalization: Taking Ningbo Digital Library Service as an Example [J]. Journal of University Library, 2011 (6): 51-54.

[3] Yan Wulin, Li Yafen. Research on the Construction Model and Operation Mechanism of Ningbo Digital Library Exploring the Innovative Way of Urban Digital Library Construction [J]. Library, 2009 (3): 84-85, 91.

[4] Zeng Zhengyu. Research on the Construction and Problem of Characteristic Resource Database--Taking the Construction of Characteristic Database of Ningbo Digital Library as an Example [J]. Library Science Research, 2013 (24): 42-47. 